\title{
Phase-dependent fluctuations of resonance fluorescence near the coherent population trapping condition
}

\author{
O. de los Santos-Sánchez 1 , 田 and H. M. Castro-Beltrán², 团 \\ ${ }^{1}$ Escuela de Ingeniería y Ciencias, Instituto Tecnológico y de Estudios Superiores de Monterrey, \\ Avenida San Carlos 100, Campus Santa Fe, Ciudad de México, 01389, México \\ ${ }^{2}$ Centro de Investigación en Ingeniería y Ciencias Aplicadas, \\ Instituto de Investigación en Ciencias Básicas y Aplicadas, Universidad Autónoma del Estado de Morelos, \\ Avenida Universidad 1001, 62209 Cuernavaca, Morelos, México
}

(Dated: February 17, 2021)

\begin{abstract}
We study phase-dependent fluctuations of the resonance fluorescence of a single $\Lambda$-type threelevel atom in the regime near coherent population trapping, i.e., alongside the two-photon detuning condition. To this end, we employ the method of conditional homodyne detection (CHD) which considers squeezing in the weak driving regime, and extends to non-Gaussian fluctuations for saturating and strong fields. In this framework, and using estimated parameter settings of the resonance fluorescence of a single trapped ${ }^{138} \mathrm{Ba}^{+}$ion, the light scattered from the probe transitions is found to manifest a non-classical character and conspicuous asymmetric third-order fluctuations in the amplitude-intensity correlation of CHD.
\end{abstract}

Keywords: resonance fluorescence, coherent population trapping, squeezing, non-Gaussian fluctuations.

\section{INTRODUCTION}

Quantum interference effects in the interaction between matter and light, epitomized by coherent population trapping (CPT) and electromagnetically induced transparency (EIT), have extensively been studied, both theoretically and experimentally, over the past decades [1, 2]. The most common level structure to enable these effects is the three-level system in the $\Lambda$ configuration $(\Lambda$ $3 \mathrm{LA})$. Although early research in this regard was primarily focused on ensembles of atomic constituents, stateof-the-art experimental developments in atomic spectroscopy have made it possible to realize EIT with a single atom in free space [3]. Indeed, these achievements have paved the way for exploring new avenues of spectroscopic analyses, besides their potential applications in the thriving field of quantum information, demonstrating, for instance, the viability of single-atom-based optical logic gates and quantum memories [4].

Both CPT and EIT are based on the cancellation of absorption when two lasers are detuned equally on adjacent transitions, thus stopping further fluorescence. Near this two-photon detuning condition, large quantum fluctuations are thus expected. Phase-sensitive fluctuations of the electromagnetic field, usually characterized by the phenomenon of squeezing, are of particular interest. Squeezing is the shrinking of a field's quadrature fluctuations at the expense of increasing those of its conjugate, and is signaled by negative spectra or variance below the shot noise level. For the resonance fluorescence of a single two-level atom, squeezing was first predicted almost forty years ago [5, 6], but it was only very recently

\footnotetext{
*Electronic address: octavio.desantos@gmail.com
}

${ }^{\dagger}$ Electronic address: hcastro@uaem.mx that squeezing of a two-level quantum dot was observed 7]. This achievement required overcoming the large collection losses of resonance fluorescence and the quantum detection losses of the standard balanced homodyne detection (BHD) technique. These issues were addressed, respectively, by the higher photon collection geometry allowed by the quantum dot, and by using a method called homodyne correlation measurement (HCM) [8 10].

The HCM method realizes an intensity-intensity correlation of the light of a previously selected quadrature; by measuring for several phases of a weak local oscillator, the method gives access to the variance (squeezing) [7] and a third-order moment of the field. The latter signals the evolution of the field after a photon was detected, as was demonstrated for the resonance fluorescence of a $\Lambda$ $3 \mathrm{LA} 11$, in a driving regime not weak enough to obtain squeezing, and far from EIT. The third-order moment is a reachable step above squeezing in the quest for highorder non-classicality [12, 13].

Conditional homodyne detection (CHD) is another measurement scheme capable of detecting phasedependent fluctuations with high efficiency owing to its conditional character $14-16$. It consists of BHD on the cue of photons recorded in a separate photodetector, giving direct access to the third-order moment of the field. Squeezing is measured if the source is weakly excited (in fact, the first motivation for the scheme) since in this case the third-order fluctuations of the field are small. However, these fluctuations, non-negligible for stronger excitation, are no less interesting: CHD goes beyond squeezing [17, 18] and reveals the non-Gaussian character of a source.

One manifestation of non-Gaussian fluctuations is the asymmetry of the field's amplitude-intensity correlation whenever two or more transitions compete [19]; it is not observed in the resonance fluorescence of a two- or threelevel atom driven by a single laser [17, 18, 20]. While this 


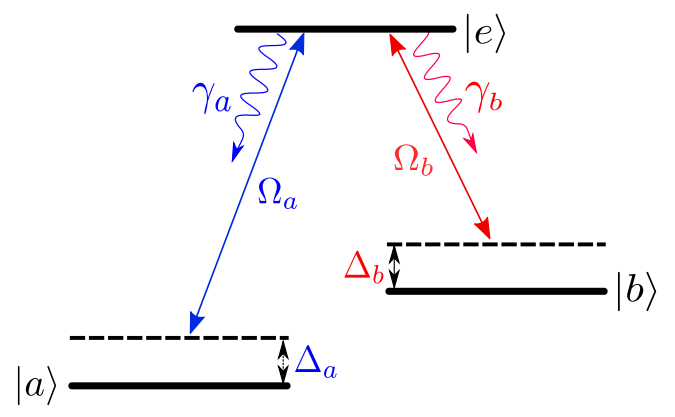

FIG. 1: Scheme of the $\Lambda$ three-level atom with spontaneous decay rates $\gamma_{a}, \gamma_{b}$, interacting with lasers with Rabi frequencies $\Omega_{a}, \Omega_{b}$ with detunings $\Delta_{a}, \Delta_{b}$.

asymmetry was readily observed for cavity QED systems both numerically [14, 19] and experimentally [15], it has been the resonance fluorescence of several 3LA systems that have provided clear theoretical access to the understanding of the asymmetry 21 26]. More recent accounts of asymmetric correlations are found in plasmonics [27] and collective cavity QED 28].

In the experiment outlined in [11], squeezing, far from the two-photon detuning, was explored in the weak field regime. In keeping with the same spirit, quantum fluctuations of the light scattered by a coherently driven V-type 3LA have thoroughly been analyzed [22]. In this work, near the two-photon detuning, we investigate, within the framework of CHD, the adjoining effect of CPT on the phase-dependent quantum fluctuations of the emitted light of the probe transition of a $\Lambda$-3LA by amplitudeintensity correlations. We follow closely the experimental conditions of observation of EIT in single ${ }^{138} \mathrm{Ba}^{+}$resonance fluorescence of Ref. [3], where saturation is present, and we find the fluctuations to be predominantly nonGaussian.

Our work is structured by introducing the atom-laser model in Section 2, discussing the role of coherent population trapping on the state populations and on the emission spectrum; section 3 is devoted to the theory of conditional homodyne detection and the analysis of quadrature fluctuations via the associated amplitude-intensity correlation. We study, in section 4, the quadrature fluctuations in the spectral domain, including squeezing and variance. Finally, in section 5, we present our conclusions and an appendix shows additional calculations.

\section{MODEL}

\section{A. Atom-Laser Interaction}

Our system, pictorially represented in Fig. 1 consists of a $\Lambda$-type three-level atom $(\Lambda$-3LA) with a single excited state $|e\rangle$ coupled by a monochromatic laser with Rabi frequency $\Omega_{a}$ to the ground state $|a\rangle$ and decay rate $\gamma_{a}$, and to a long-lived state $|b\rangle$ by a monochromatic laser with Rabi frequency $\Omega_{b}$ and decay rate $\gamma_{b}$. Decay from $|b\rangle$ to $|a\rangle$ is dipole-forbidden. Henceforth, the fields driving the $|a\rangle \rightarrow|e\rangle$ and $|b\rangle \rightarrow|e\rangle$ transitions will be referred to as the probe and control fields. We define the atomic operators as $\hat{\sigma}_{j k}=|j\rangle\langle k|$.

Under the above considerations, the system's evolution, in free space, and in the frame rotating at the laser frequencies, $\nu_{a}$ and $\nu_{b}$, is governed by the master equation $\dot{\tilde{\rho}}(t)=-i[\hat{H}, \tilde{\rho}]+\sum_{j} \frac{\gamma_{j}}{2} \mathcal{L}_{\hat{\sigma}_{j e}}[\tilde{\rho}]$, in which

$$
\hat{H}=\sum_{j=a, b}-\Delta_{j} \hat{\sigma}_{j j}+\frac{\Omega_{j}}{2}\left(\hat{\sigma}_{e j}+\hat{\sigma}_{j e}\right)
$$

is the atom-laser Hamiltonian, and $\Delta_{j}=\omega_{e j}-\nu_{j}$ labels the individual atom-laser detunings. Dissipation is accounted for by the action of the Lindblad generator $\mathcal{L}_{\hat{O}}[\tilde{\rho}]=2 \hat{O} \tilde{\rho} \hat{O}^{\dagger}-\hat{O}^{\dagger} \hat{O} \tilde{\rho}-\tilde{\rho} \hat{O}^{\dagger} \hat{O}$, with $\hat{O}=\hat{\sigma}_{j e}$. With the help of the relationship $\hat{\sigma}_{j k} \hat{\sigma}_{l m}=\hat{\sigma}_{j m} \delta_{k l}$, the master equation can be explicitly recast as

$$
\dot{\tilde{\rho}}(t)=-i[\hat{H}, \tilde{\rho}]+\sum_{j=a, b} \gamma_{j} \tilde{\rho}_{e e} \hat{\sigma}_{j j}-\frac{\gamma_{j}}{2}\left(\hat{\sigma}_{e e} \tilde{\rho}+\tilde{\rho} \hat{\sigma}_{e e}\right)
$$

With the relation $\left\langle\hat{\sigma}_{j k}\right\rangle=\tilde{\rho}_{k j}$, Eq. (2) allows us to arrive at the following set of linear equations for populations:

$$
\begin{aligned}
\left\langle\dot{\hat{\sigma}}_{a a}\right\rangle= & -i \frac{\Omega_{a}}{2}\left(\left\langle\hat{\sigma}_{a e}\right\rangle-\left\langle\hat{\sigma}_{e a}\right\rangle\right)+\gamma_{a}\left\langle\hat{\sigma}_{e e}\right\rangle \\
\left\langle\dot{\hat{\sigma}}_{b b}\right\rangle= & -i \frac{\Omega_{b}}{2}\left(\left\langle\hat{\sigma}_{b e}\right\rangle-\left\langle\hat{\sigma}_{e b}\right\rangle\right)+\gamma_{b}\left\langle\hat{\sigma}_{e e}\right\rangle \\
\left\langle\dot{\hat{\sigma}}_{e e}\right\rangle= & i \frac{\Omega_{a}}{2}\left(\left\langle\hat{\sigma}_{a e}\right\rangle-\left\langle\hat{\sigma}_{e a}\right\rangle\right)+i \frac{\Omega_{b}}{2}\left(\left\langle\hat{\sigma}_{b e}\right\rangle-\left\langle\hat{\sigma}_{e b}\right\rangle\right) \\
& -\gamma\left\langle\hat{\sigma}_{e e}\right\rangle
\end{aligned}
$$

with $\gamma=\gamma_{a}+\gamma_{b}$, and coherences:

$$
\begin{aligned}
\left\langle\dot{\hat{\sigma}}_{a b}\right\rangle= & i \frac{\Omega_{a}}{2}\left\langle\hat{\sigma}_{e b}\right\rangle-i \frac{\Omega_{b}}{2}\left\langle\hat{\sigma}_{a e}\right\rangle-i\left(\Delta_{a}-\Delta_{b}\right)\left\langle\hat{\sigma}_{a b}\right\rangle, \\
\left\langle\dot{\hat{\sigma}}_{a e}\right\rangle= & i \frac{\Omega_{a}}{2}\left(\left\langle\hat{\sigma}_{e e}\right\rangle-\left\langle\hat{\sigma}_{a a}\right\rangle\right)-i \frac{\Omega_{b}}{2}\left\langle\hat{\sigma}_{a b}\right\rangle \\
& -\left(\frac{\gamma}{2}+i \Delta_{a}\right)\left\langle\hat{\sigma}_{a e}\right\rangle \\
\left\langle\dot{\hat{\sigma}}_{b e}\right\rangle= & -\frac{i \Omega_{a}}{2}\left\langle\hat{\sigma}_{b a}\right\rangle+\frac{i \Omega_{b}}{2}\left(\left\langle\hat{\sigma}_{e e}\right\rangle-\left\langle\hat{\sigma}_{b b}\right\rangle\right) \\
& -\left(\frac{\gamma}{2}+i \Delta_{b}\right)\left\langle\hat{\sigma}_{b e}\right\rangle \\
\left\langle\dot{\hat{\sigma}}_{j k}\right\rangle= & \left\langle\dot{\hat{\sigma}}_{k j}\right\rangle^{*}
\end{aligned}
$$

The solution to these equations (a set of nine Bloch equations) is to be obtained numerically and their structure will facilitate the assessment of the sought correlation functions via the quantum regression formula, combined with the employment of matrix methods. For later use, we define the values of the atomic operators in the steady state as

$$
\left\langle\hat{\sigma}_{j k}(t \rightarrow \infty)\right\rangle=\left\langle\hat{\sigma}_{j k}\right\rangle_{s s}=\alpha_{j k}
$$



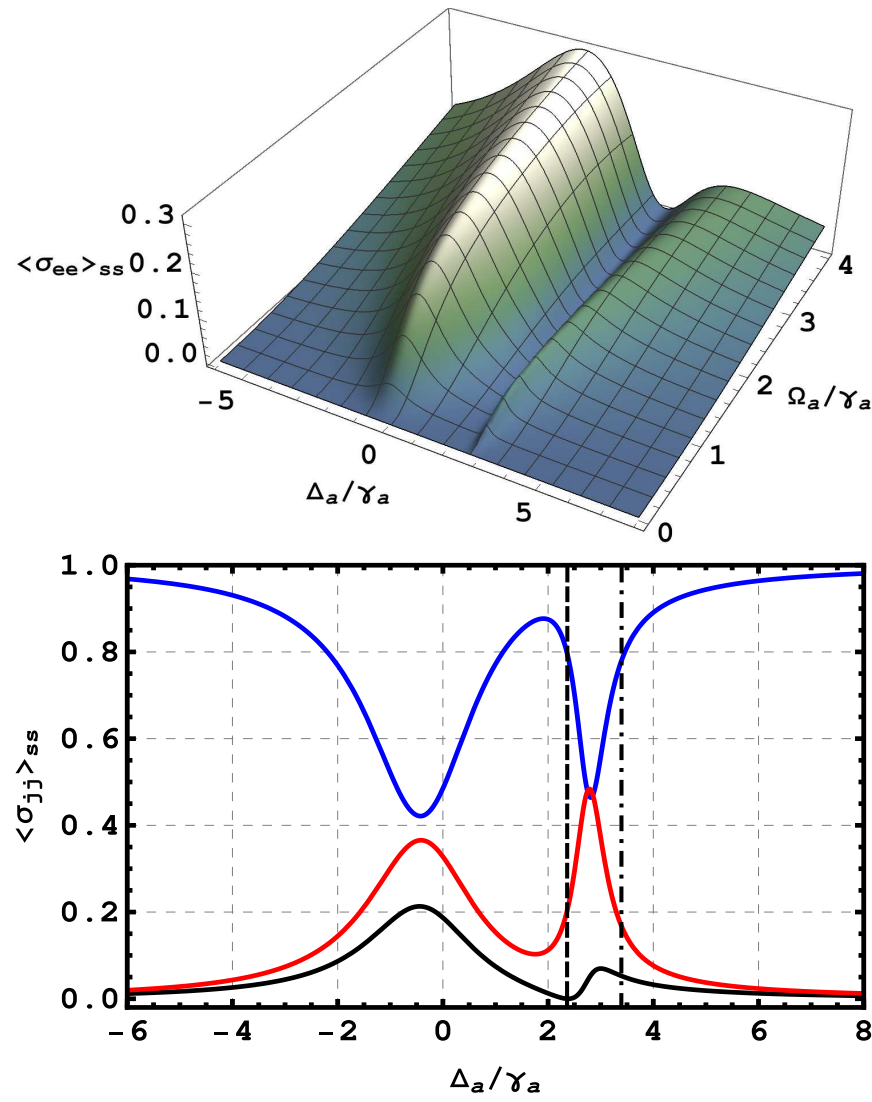

FIG. 2: Upper panel: Occupation probability of the excited state, $\left\langle\sigma_{e e}\right\rangle_{s s}$, versus the scaled detuning $\Delta_{a} / \gamma_{a}$ and Rabi frequency $\Omega_{a} / \gamma_{a}$. Lower panel: Populations $\left\langle\sigma_{j j}\right\rangle_{s s}$, for $j=$ $a, b$ and $e$ (blue, red and black lines, respectively) as functions of $\Delta_{a} / \gamma_{a}$; the black curve is the corresponding cross sectional profile of the upper figure at $\Omega_{a} / \gamma_{a} \approx 1.12$. The remaining parameters are $\Omega_{b} / \gamma_{a} \approx 2.15$ and $\Delta_{b} / \gamma_{a} \approx 2.38$.

Besides, in order for our findings to be possibly put to the test in a given realization, we shall consider the decays $\gamma_{a}=14.7 \mathrm{MHz}$ and $\gamma_{b}=5.4 \mathrm{MHz}$, observed in ${ }^{138} \mathrm{Ba}^{+}$ions [3, 11, 29]. Although a more accurate description of Barium resonance fluorescence would entail considering its multilevel structure, being composed of eight energy levels, it suffices for our purposes to deal with the simplified three-level system as a proxy for specifying the relevant allowed dipole transitions that take part in the dynamics. Parenthetically, the isolation of a single three-level configuration can be implemented through a proper optical pumping arrangement.

\section{B. Role of Coherent Population Trapping}

The $\Lambda$-type three-level atom is an archetypal system that readily fulfills the necessary conditions for coherent population trapping (CPT) to take place [1, 2]. In such a scenario, the system is known to evolve towards the trapping state $|u\rangle=\left(\Omega_{b}|a\rangle-\Omega_{a}|b\rangle\right) / \sqrt{\Omega_{a}^{2}+\Omega_{b}^{2}}$ that turns out to be decoupled from the lasers, thereby dropping the long-term excited-state population $\alpha_{e e}$ to nearly zero. The manifestation of this effect is exemplified in the upper panel of Fig. 2 where the steady state population of the excited state is shown as a function of both the detuning and Rabi frequency of the probe laser $(e \rightarrow a$ transition); the values of the parameters associated with the control field are, henceforth, taken to be fixed and the same as those reported in [3], namely, $\Omega_{b} / \gamma_{a} \approx 2.15$ and $\Delta_{b} / \gamma_{a} \approx 2.38$. In accord with the well-established prescription to determine the frequency region around which the atom is essentially transparent to the incoming probe field, the so-called Raman resonance condition, the probe detuning must be such that $\Delta_{a} \approx \Delta_{b}$ is satisfied; the role of the probe intensity $\Omega_{a}$ is that of slightly modifying the width of such a transparent frequency window. Its location is also depicted in the lower panel of Fig. 2 showing the cross sectional profile of the upper figure (black line) at $\Omega_{a} / \gamma_{a} \approx 1.12$ where, in turn, we can observe the complete depopulation of the excited state at $\Delta_{a} / \gamma_{a} \approx 2.38$ (dashed vertical line); the populations of the $|a\rangle$ and $|b\rangle$ states are also added as a supplementary view of their behavior as functions of the probe detuning.

The foregoing was not the actual condition under which the experiments [3] were performed, but instead the detuning was chosen so as to fit the value of the corresponding saturation parameter, $\Omega_{j}^{2} /\left(\gamma_{j}^{2}+\Delta_{j}^{2}\right)$, and taken to be $\sim 0.1$ for the $a \rightarrow e$ transition. This choice gives rise to a detuning of about $\Delta_{a} / \gamma_{a} \approx 3.4$, the location of which being also indicated in the figure (dotted-dashed vertical line); the saturation parameter associated with the control field was set to 0.8 . So, for this particular choice of probe and control detunings that drive the $a \rightarrow e$ transition out of the Raman resonance condition, the complete depopulation of the $|e\rangle$ state can be avoided or delayed, a working situation that will permit us to study the nonclassical properties of the scattered light we seek to assess. It is worth commenting that if, instead, the $a \rightarrow e$ transition were driven more strongly than the $b \rightarrow e$ one, such that $\Omega_{a}>\Omega_{b}$, for general detunings, the population would end up in the $|b\rangle$ state, with $\Omega_{b} \ll \gamma_{a}$. So then, the strong transition would be turned off due to lack of recycling population to $|e\rangle$.

So, having established the present configuration of laser intensities and frequencies, we find it pertinent, at this stage, to depict the stationary power spectrum of the re-emitted light obtained by use of the WienerKhintchine formula

$$
S(\omega)=\frac{1}{\pi \alpha_{e e}} \operatorname{Re} \int_{0}^{\infty} d \tau e^{-i \omega \tau}\left\langle\hat{\sigma}_{e a}(0) \hat{\sigma}_{a e}(\tau)\right\rangle_{s s},
$$

i.e., the Fourier transform of the autocorrelation function of the dipole field, $\left\langle\hat{\sigma}_{e a}(0) \hat{\sigma}_{a e}(\tau)\right\rangle_{s s}$, where $s s$ indicates that the process is stationary; the prefactor $\left(\pi \alpha_{e e}\right)^{-1}$ normalizes the integral over all frequencies. For convenience, the spectrum is separated into its coherent and 


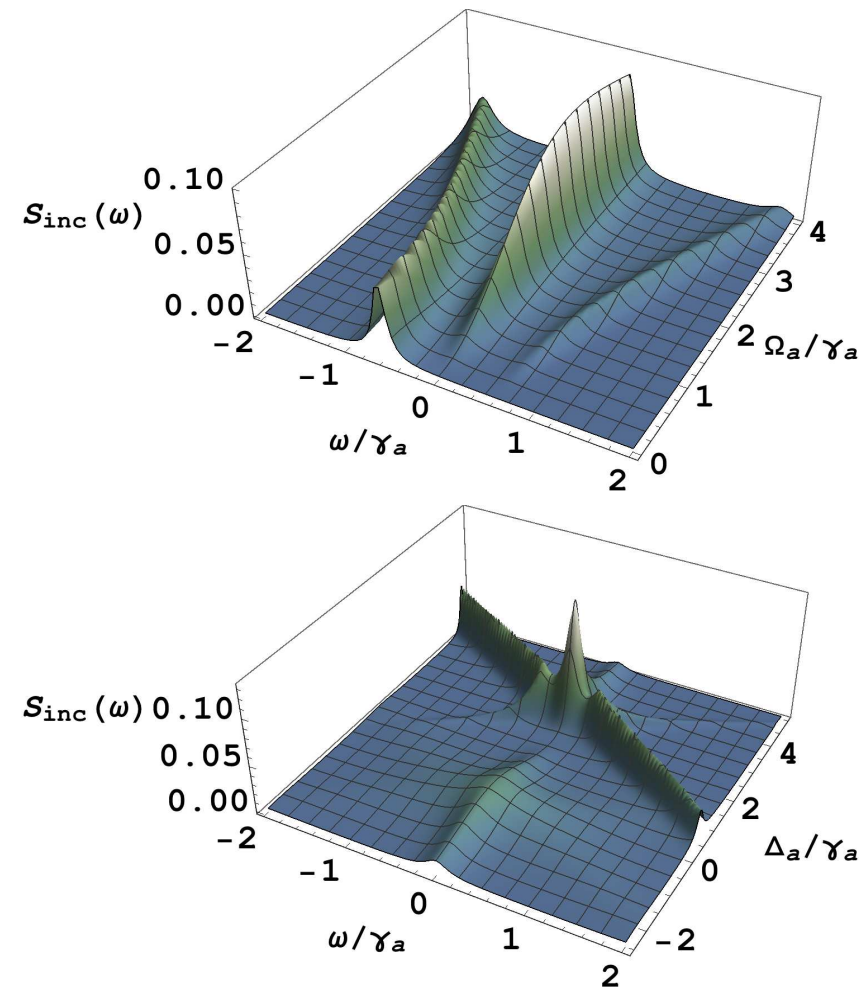

FIG. 3: Incoherent spectrum, $S_{\text {inc }}(\omega)$, of the $e \rightarrow a$ transition as a function of the scaled probe laser intensity $\Omega_{a} / \gamma_{a}$ (upper panel, for $\Delta_{a} / \gamma_{a} \approx 3.4$ ) and the detuning $\Delta_{a} / \gamma_{a}$ (lower panel, for $\Omega_{a} / \gamma_{a} \approx 1.12$ ); the range of spectral distribution is also displayed in units of $\gamma_{a}$. The remaining parameters are the same as in Fig. 2.

incoherent parts, namely, $S(\omega)=S_{c o h}(\omega)+S_{\text {inc }}(\omega)$, as a result of considering the dynamics of the atomic variables to be split into their mean and fluctuations, viz. $\hat{\sigma}_{j k}(t)=\alpha_{j k}+\Delta \hat{\sigma}_{j k}(t)$, with $\left\langle\Delta \hat{\sigma}_{j k}(t)\right\rangle=0$. In doing so, we get

$$
S_{c o h}(\omega)=\frac{\left|\alpha_{e a}\right|^{2}}{\pi \alpha_{e e}} \operatorname{Re} \int_{0}^{\infty} d \tau e^{-i \omega \tau}=\frac{\left|\alpha_{e a}\right|^{2}}{\pi \alpha_{e e}} \delta(\omega),
$$

where $\alpha_{e a}=\left\langle\hat{\sigma}_{e a}\right\rangle_{s s}$, and

$$
S_{i n c}(\omega)=\frac{1}{\pi \alpha_{e e}} \operatorname{Re} \int_{0}^{\infty} d \tau e^{-i \omega \tau}\left\langle\Delta \hat{\sigma}_{e a}(0) \Delta \hat{\sigma}_{a e}(\tau)\right\rangle_{s s}
$$

the former being the coherent constituent of the spectrum owing to elastic scattering, and the latter the incoherent part of the spectrum that is brought about by atomic fluctuations. The main features of the $\Lambda$-type three-level atom spectrum have already been studied from the weak to the strong field limit, both theoretically and experimentally [30]. Figure 3 shows a three dimensional view of the incoherent part of the spectrum associated with the $e \rightarrow a$ transition, the one of interest to us, as a function of the probe intensity (upper panel) and the detuning (lower panel); details of the steps involved in the calculations herein via the matrix analysis are included in Ap-

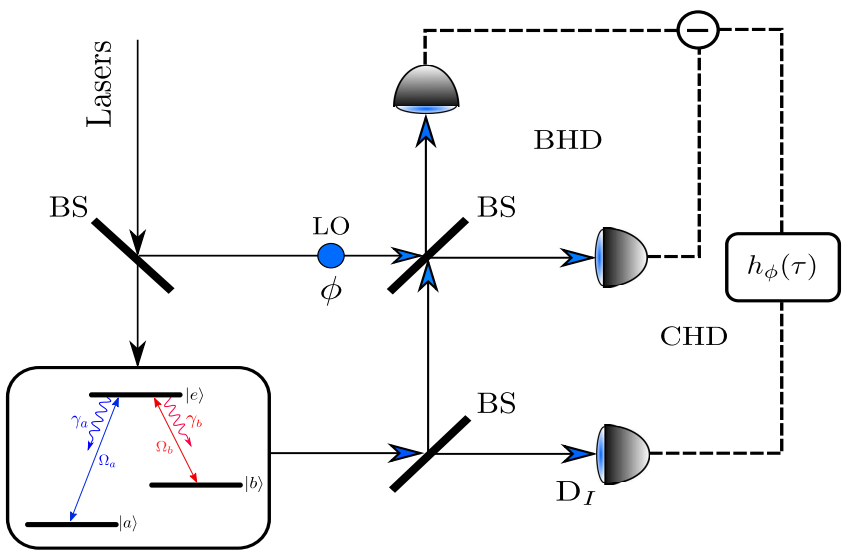

FIG. 4: Scheme of conditional homodyne detection (CHD). It features a balance homodyne detection setup to assess the quadrature of the field on the condition of photon detection via detector $\mathrm{D}_{I}$. BS and LO stand for beam splitter and local oscillator, respectively.

pendix $\mathrm{A}$ The general spectral profile can be understood in terms of dressed-state configuration that follows from properly diagonalizing the atom-field Hamiltonian [31], emphasizing the fact that, above saturation, the spectrum displays the appearance of Rabi sidebands as the intensity field increases, as one can see in the upper panel of the figure. By setting the Rabi frequency at, say, $\Omega_{a} / \gamma_{a}=1.12$, such sidebands become sufficiently conspicuous and the dependency of their profile upon the detuning is shown in the lower panel.

\section{AMPLITUDE-INTENSITY CORRELATION}

In this section we present the theory of conditional homodyne detection (CHD) in order to assess and discuss the time-asymmetry, the non-Gaussianity and the nonclassicality of the light scattered from the atomic system under study. In the next section we move to the frequency domain. The CHD setup is sketched in Fig. 4. In one arm of the setup a quadrature of the source light, $E_{\phi} \propto \hat{\sigma}_{\phi}=\left(\hat{\sigma}_{e a} e^{-i \phi}+\hat{\sigma}_{a e} e^{i \phi}\right) / 2$, is analyzed in balanced homodyne detection (BHD), where $\phi$ is the phase of the local oscillator (LO). This signal has a delay $\tau$ with respect to the measurement of the source's intensity in another arm, proportional to the excited-state population, $I \propto\left\langle\hat{\sigma}_{e a} \hat{\sigma}_{a e}\right\rangle=\left\langle\hat{\sigma}_{e e}\right\rangle$. Thus, the outcome is an amplitude-intensity correlation that reads

$$
h_{\phi}(\tau)=\frac{\left\langle: \hat{\sigma}_{e a}(0) \hat{\sigma}_{a e}(0) \hat{\sigma}_{\phi}(\tau):\right\rangle_{s s}}{\alpha_{e e} \alpha_{\phi}}
$$

where the dots :: indicate normal and time operator ordering, and $\alpha_{\phi}=\left(\alpha_{e a} e^{-i \phi}+\alpha_{a e} e^{i \phi}\right) / 2$ is the stationary value of the quadrature amplitude. 


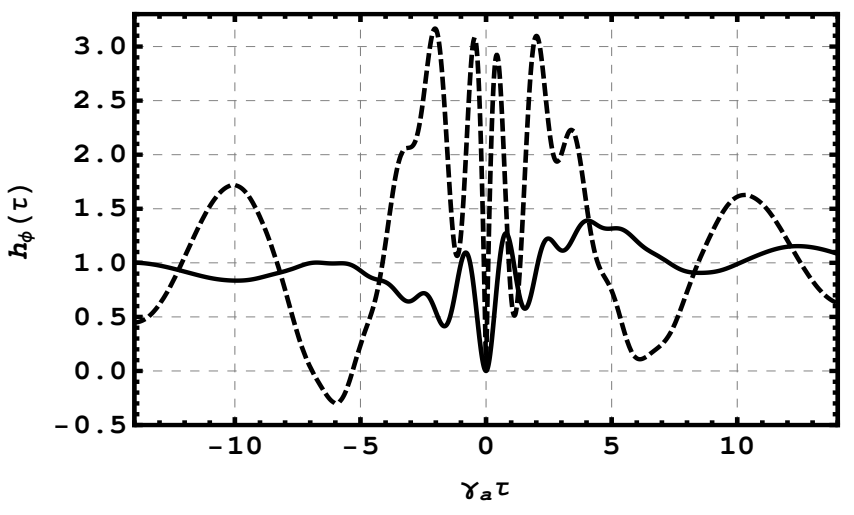

FIG. 5: Amplitude-intensity correlations of light from the $e \rightarrow a$ transition, as a function of the scaled time $\gamma_{a} \tau$, for $\phi=0$ (continuous line) and $\phi=\pi / 2$ (dotted-dashed line). The parameters are: $\Omega_{a} \approx 1.12 \gamma_{a}, \Omega_{b} \approx 2.15 \gamma_{a} \Delta_{a}=3.40 \gamma_{a}$, $\Delta_{b}=2.38 \gamma_{a}$.

\section{A. Time-asymmetry and Non-Gaussianity}

Resonance fluorescence is a highly non-linear process, preventing its description in terms of quasi-probability distributions, i.e., it does not admit a Fokker-Planck type of equation. The non-linearity leads to nonGaussian fluctuations, thus giving rise to non-vanishing odd-order moments. Autocorrelation functions such as that for the spectrum, $\left\langle\sigma_{e a}(0) \sigma_{a e}(\tau)\right\rangle$; squeezing, $\left\langle\Delta \sigma_{\phi}(0) \Delta \sigma_{\phi}(\tau)\right\rangle$; and photon-photon correlation, $\left\langle\sigma_{e a}(0) \sigma_{e a}(\tau) \sigma_{a e}(\tau) \sigma_{a e}(0)\right\rangle$, are of even-order and, as such, time-symmetric [19]. These functions do not address the non-Gaussianity of the field's fluctuations.

In amplitude-intensity correlations, Eq.(14), on the other hand, such a symmetry is not guaranteed: being different observables, the outcome will be dependent on the time order of measurements. For instance, the quadrature is measured (preselected) for $\tau \geq 0$ and the intensity for $\tau \leq 0$ (quadrature is post-selected), a process in which time-asymmetry is expected to be revealed. Moreover, this correlation would allow us to explore non-classical features of light beyond squeezing and antibunching.

Applying the time and normal operator orderings in Eq. (14) we arrive at the following expressions for positive and negative time intervals,

$$
\begin{aligned}
& h_{\phi}(\tau \geq 0)=\frac{\left\langle\hat{\sigma}_{e a}(0) \hat{\sigma}_{\phi}(\tau) \hat{\sigma}_{a e}(0)\right\rangle_{s s}}{\alpha_{e e} \alpha_{\phi}} \\
& h_{\phi}(\tau \leq 0)=\frac{\operatorname{Re}\left[e^{-i \phi}\left\langle\hat{\sigma}_{e a}(0) \hat{\sigma}_{e e}\left(\tau_{-}\right)\right\rangle_{s s}\right]}{\alpha_{e e} \alpha_{\phi}}
\end{aligned}
$$

The asymmetry in time revealed by CHD, as shown in Fig. 5, is an indicative of non-Gaussian noise. The correlation (14) contains a product of three dipole operators or, more generally, three field amplitude operators. This means that $h_{\phi}(\tau)$ provides access up to third order fluctuations; since these are non-Gaussian, this third-order correlation does not vanish. To better distinguish the asymmetry and the size of these fluctuations, we proceed, as we did with the spectrum, to split the dipole dynamics into its mean plus fluctuations, $\hat{\sigma}_{j k}=\alpha_{\phi}+\Delta \hat{\sigma}_{j k}$ [17],

$$
h_{\phi}(\tau)=1+h_{\phi}^{(2)}(\tau)+h_{\phi}^{(3)}(\tau)
$$

where

$$
h_{\phi}^{(2)}(\tau)=\frac{\left\langle:\left[\alpha_{e a} \Delta \hat{\sigma}_{a e}(0)+\alpha_{a e} \Delta \hat{\sigma}_{e a}(0)\right] \Delta \hat{\sigma}_{\phi}(\tau):\right\rangle_{s s}}{\alpha_{e e} \alpha_{\phi}},
$$

and

$$
h_{\phi}^{(3)}(\tau)=\frac{\left\langle: \Delta \hat{\sigma}_{e a}(0) \Delta \hat{\sigma}_{a e}(0) \Delta \hat{\sigma}_{\phi}(\tau):\right\rangle_{s s}}{\alpha_{e e} \alpha_{\phi}},
$$

are the components of, respectively, second- and thirdorder in the dipole fluctuations of $h_{\phi}(\tau)$, where $\Delta \hat{\sigma}_{\phi}=$ $\left(\Delta \hat{\sigma}_{e a} e^{-i \phi}+\Delta \hat{\sigma}_{a e} e^{i \phi}\right) / 2$ is the quadrature fluctuation operator. Fluctuations are said to be Gaussian if $h_{\phi}^{(3)}(\tau) \rightarrow$ 0 , which can occur when the transition is weakly driven.

For positive time intervals between photon and quadrature detections, we get

$$
\begin{aligned}
& h_{\phi}^{(2)}(\tau \geq 0)=\frac{2 \operatorname{Re}\left[\alpha_{a e}\left\langle\Delta \hat{\sigma}_{e a}(0) \Delta \hat{\sigma}_{\phi}(\tau)\right\rangle_{s t}\right]}{\alpha_{e e} \alpha_{\phi}} \\
& h_{\phi}^{(3)}(\tau \geq 0)=\frac{\left\langle\Delta \hat{\sigma}_{e a}(0) \Delta \hat{\sigma}_{\phi}(\tau) \Delta \hat{\sigma}_{a e}(0)\right\rangle_{s t}}{\alpha_{e e} \alpha_{\phi}} .
\end{aligned}
$$

We show in Fig. 6 the foregoing second- and third-order correlations. For both quadratures, the third-order constituent (blue lines) represents the main contribution, almost that of the total $h_{\phi}(\tau)$ (black lines). This is understandable from the fact that we are above the saturation threshold [22], a regime where non-Gaussian fluctuations become significant. In this regime the dipole $\alpha_{e a}$, indicative of the coherence induced by the laser, is small; most of the total emission is incoherent. This observation can be quantitatively revealed from the fact that $h_{\phi}(0)=0$ (just as it occurs for photon correlations in resonance fluorescence), which leads to the relation 22]

$$
h_{\phi}^{(3)}(0)=-\left[1+h_{\phi}^{(2)}(0)\right]=\frac{2\left(\left|\alpha_{e a}\right|^{2}-\alpha_{e e}\right)}{\alpha_{e e}} .
$$

For strong fields, $\left|\alpha_{e a}\right| \ll \alpha_{e e}, h_{\phi}^{(3)}(0)$ reaches its extremal value -2 , thereby making the dipole factor in Eq. (20) small compared to the third-order term.

Even though the splitting itself cannot be directly realizable from the experimental viewpoint via the measurement scheme, it provides us with valuable theoretical information to be able to discern the actual contribution to the system's fluctuations.

For $\tau<0$, we want to stress the fact that the outcome of CHD correlation should be taken with special care: it is to be interpreted as the measurement of the intensity 

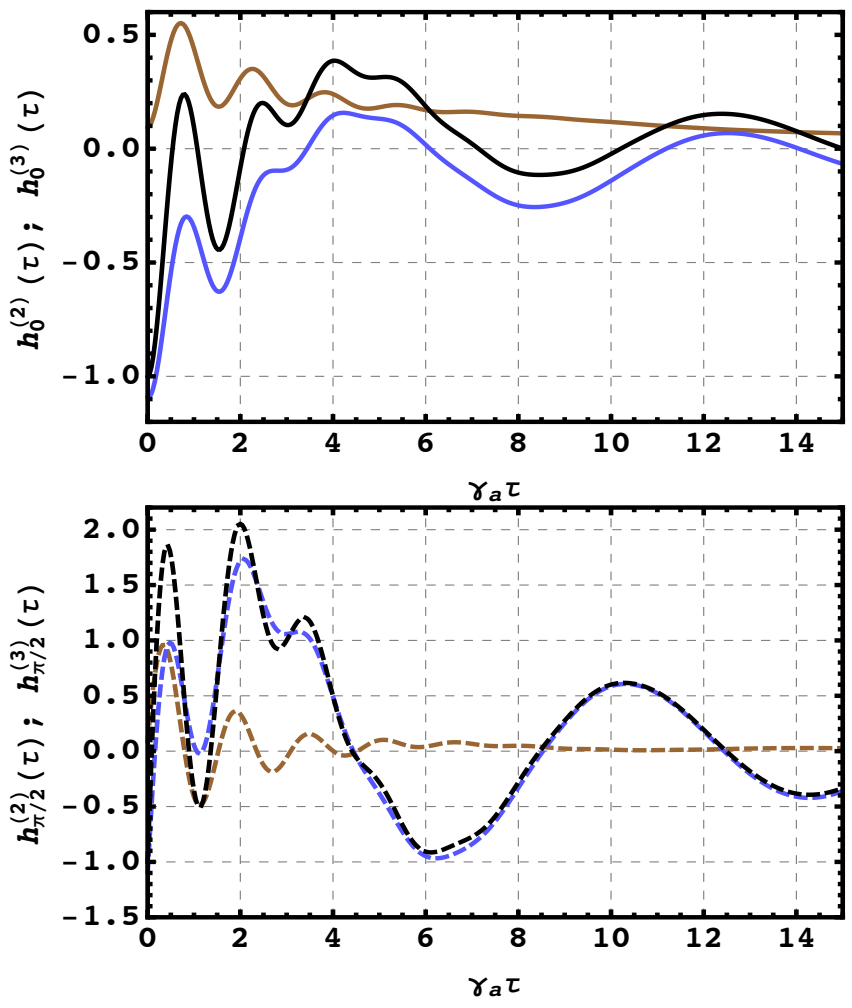

FIG. 6: Splitting of the intensity-field correlations shown in Fig. 5 into their second- $\left(h_{\phi}^{(2)}(\tau)\right.$, brown line) and third-order $\left(h_{\phi}^{(3)}(\tau)\right.$, blue line) constituents, for $\phi=0$ (upper panel) and $\phi=\pi / 2$ (lower panel). For comparison, in both cases the black curve represents the sum $h_{\phi}^{(2)}(\tau)+h_{\phi}^{(3)}(\tau)$. The parameters are the same as those of Fig. 5 .

after the detection of the amplitude. Thus, as previously underlined, the asymmetry results from the different fluctuations of the light's amplitude and intensity. Time and normal operator ordering leads to

$$
h_{\phi}(\tau \leq 0)=1+\frac{\operatorname{Re}\left[e^{-i \phi}\left\langle\Delta \hat{\sigma}_{e a}(0) \Delta \hat{\sigma}_{e e}(|\tau|)\right\rangle_{s t}\right]}{\alpha_{e e} \alpha_{\phi}},
$$

i. e., the correlation is only of second order in the dipole fluctuations, albeit with $\Delta \hat{\sigma}_{e e}$ instead of the quadrature amplitude $\Delta \hat{\sigma}_{\phi}$ fluctuation operator.

\section{B. Non-classicality}

The initial motivation for CHD was to detect squeezing from weak sources, such as cavity QED [14, 15]. Resonance fluorescence is also a producer of weakly squeezed light [5, 6]. In order to produce light in a squeezed state, a non-classical property of light, these sources must be weakly driven, so that the third-order fluctuations discussed above are small. As we will see later, the remaining second-order signal is related to the spectrum of squeezing. CHD, hence, gives non-classical criteria in the time domain as resulting of violation of the classical inequalities [14, 15]

$$
\begin{aligned}
0 \leq h_{\phi}(\tau)-1 & \leq 1, \\
\left|h_{\phi}^{(2)}(\tau)\right| \leq\left|h_{\phi}^{(2)}(0)\right| & \leq 1,
\end{aligned}
$$

where the second relation is derived for Gaussian fluctuations. More recently, it was found that light in a coherent state obeys [22]

$$
-1 \leq h_{\phi}(\tau) \leq 1
$$

light outside these bounds violates Poissonian statistics. According to these criteria, we see in Figs. 5 and 6 that both the in-phase $(\phi=0$, continuous line) and out-ofphase $(\phi=\pi / 2$, dashed line) quadratures of the field display a non-classical character, violating one or more inequalities. The fact that $h_{\phi}(0)=0$ already shows a non-classical feature, akin to antibunching in the intensity fluctuations. Also, moderately strong fields easily drive $h_{\phi}(\tau)$ out of the classical bounds.

We see, then, that CHD clearly reveals non-classicality of quadratures in the time domain. Let us now proceed to scrutinize the spectral profile of amplitude-intensity correlations in the frequency domain.

\section{QUADRATURE SPECTRA}

Since in CHD the signal is time-asymmetric, carrying different information for positive and negative intervals, the spectra of quadratures measured from the amplitudeintensity correlation should be calculated separately [22]:

$$
\begin{gathered}
S_{\phi}^{(\tau \geq 0)}(\omega)=4 \gamma_{a} \alpha_{e e} \int_{0}^{\infty} d \tau \cos \omega \tau\left[h_{\phi}(\tau \geq 0)-1\right], \\
S_{\phi}^{(\tau \leq 0)}=4 \gamma_{a} \alpha_{e e} \int_{-\infty}^{0} d \tau \cos (\omega \tau)\left[h_{\phi}(\tau \leq 0)-1\right],
\end{gathered}
$$

for positive and negative time intervals, respectively. The prefactor $\gamma_{a} \alpha_{e e}$ is the photon emission rate in the probe transition. In Fig. 7 we show the spectra calculated from Eqs. (27) and (28) for both quadratures and the same parameter values of Fig. 5 . From the CHD viewpoint, negative values of the spectrum are signature of non-classical scattered light beyond squeezing, which is confirmed for both quadratures, with the $\pi / 2$ quadrature exhibiting a more pronounced non-classical behavior than the other. Fig. [ 8 also shows the overall spectral profile as a function of the probe laser's Rabi frequency for the $\pi / 2$ quadrature only, and for positive (upper panel) and negative (lower panel) intervals. This more complete landscape allows us to verify non-classicality of light revealed by clear-cut negative valleys even for excitation above saturation.

Following the splitting of $h_{\phi}(\tau \geq 0)$, Eq. (17), the spectra of second- and third-order dipole fluctuations are

$$
S_{\phi}^{(N)}(\omega)=4 \gamma_{a} \alpha_{e e} \int_{0}^{\infty} d \tau \cos \omega \tau h_{\phi}^{(N)}(\tau),
$$



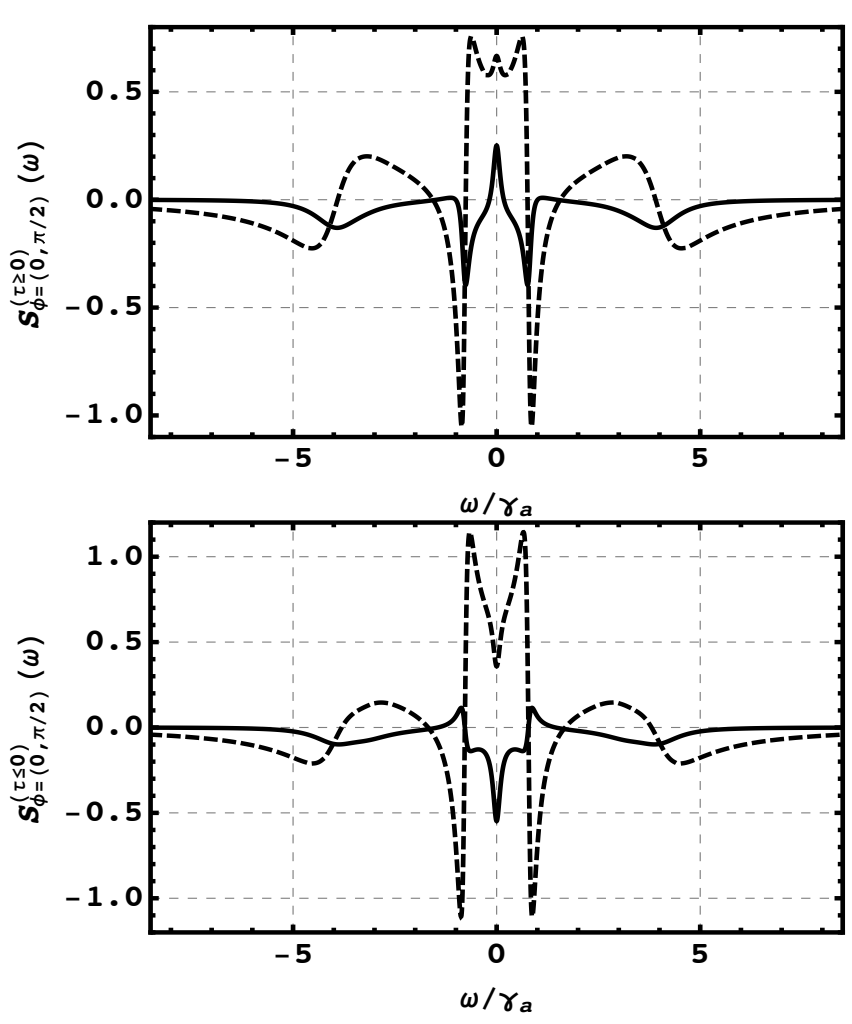

FIG. 7: Spectra, Eqs. (27) and (28), upper and lower panels, respectively, for $\phi=0$ (continuous line) and $\pi / 2$ (dashed line). The parameters are the same as those of Fig. 5$]$

for $N=2,3$, so that $S_{\phi}^{(\tau \geq 0)}(\omega)=S_{\phi}^{(2)}(\omega)+S_{\phi}^{(3)}(\omega)$. These are shown in Fig. 9 for both quadratures, corresponding to the CHD signals of Fig. 6. We find that the second-order spectra are mostly positive, while the thirdorder spectrum is negative for $\phi=0$, there are negative bands for $\phi=\pi / 2$. In Fig. 10 the dependence of $S_{\pi / 2}^{(N)}$ on the detuning of the probe laser is shown. A quite similar spectral landscape (not shown) was found in the secondorder correlation for $\tau \leq 0$, Eq. (28). The lineshapes are very complicated, but the dispersive features at the sides reveal the non-Gaussianity of the field [20, 22].

The above spectra clearly deviate from the more conventional measure of non-classical phase-dependent fluctuations, squeezing, due to the non-linearity induced by the strong lasers. Understood operationally as the reduction of quantum fluctuations below the shot noise limit, squeezing can be obtained in the spectral domain as the Fourier transform of symmetric photocurrent fluctuations in homodyne detection 33]. For our source,

$$
\begin{aligned}
S_{\phi}(\omega)= & 8 \gamma_{a} \eta \int_{0}^{\infty} d \tau \cos \omega \tau\left\langle: \Delta \hat{\sigma}_{\phi}(0) \Delta \hat{\sigma}_{\phi}(\tau):\right\rangle_{s s} \\
= & 8 \gamma_{a} \eta \int_{0}^{\infty} d \tau \cos \omega \tau \\
& \times \operatorname{Re}\left[e^{-i \phi}\left\langle\Delta \hat{\sigma}_{e a}(0) \Delta \hat{\sigma}_{\phi}(\tau)\right\rangle_{s s}\right]
\end{aligned}
$$

where $\eta$ is a combined collection and detection efficiency,
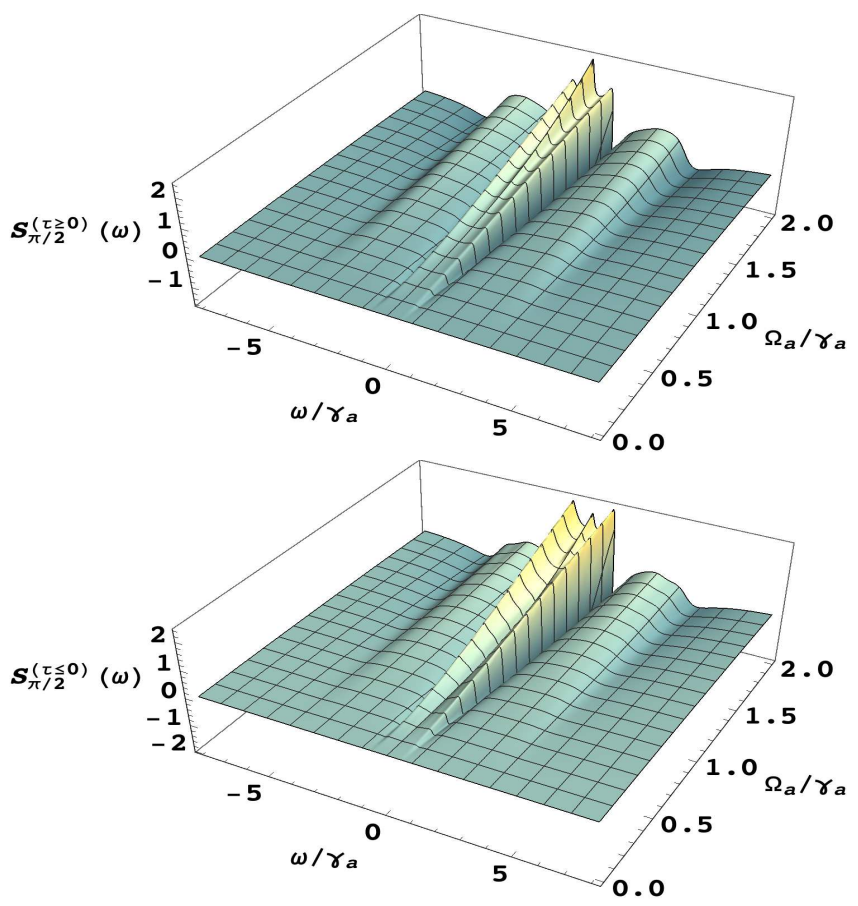

FIG. 8: Fourier cosine transform of $h_{\pi / 2}^{(N)}(\tau)$, for $\tau \geq 0$ (upper panel) and $\tau \leq 0$ (lower panel), as a function of the scaled Rabi frequency $\Omega_{a} / \gamma_{a}$, for $\Omega_{b} \approx 2.15 \gamma_{a} \Delta_{a}=3.4 \gamma_{a}$ and $\Delta_{b}=$ $2.38 \gamma_{a}$.

and the dots :: state that the operators must follow time and normal orderings. It was shown in [14, 15] that, in the weak-field limit, when third-order fluctuations can be neglected, the second-order spectrum from CHD, from Eqs. (29) and (20), is indeed the spectrum of squeezing, but unafected by detector losses, i.e., $S_{\phi}^{(2)}(\omega)=S_{\phi}(\omega) / \eta$, owing to the conditional character of CHD.

Figure 11 displays a 3D plot of the spectrum of squeezing, given by Eq. (30) with $\eta=1$, as a function of the probe laser intensity $\Omega_{a} / \gamma_{a}$ (upper panel) and detuning $\Delta_{a} / \gamma_{a}$ (lower panel), for the $\phi=\pi / 2$ quadrature. The figure shows up indicatives of squeezing (negative values on the spectral content) for a moderate detuning, at $\Delta_{a} / \gamma_{a}=2.38$, around which CPT takes place, even for laser intensities above saturation. A slightly higher degree of squeezing comes about within certain regions of the spectrum by fixing the laser intensity, at $\Omega_{a} / \gamma_{a}=0.1$, say, and varying the detuning (see lower panel).

An alternative picture of squeezing is the variance

$$
V_{\phi}=\left\langle:\left(\Delta \sigma_{\phi}\right)^{2}:\right\rangle_{s t}=\operatorname{Re}\left[e^{-i \phi}\left\langle\Delta \sigma_{e g} \Delta \sigma_{\phi}\right\rangle_{s s}\right],
$$

related to the integrated spectrum as $\int_{-\infty}^{\infty} S_{\phi}(\omega) d \omega=$ $4 \pi \gamma_{a} \eta V_{\phi}$. This quantity is depicted in Fig. 12 for $\phi=\pi / 2$ as a function of the scaled detuning and laser intensity, revealing a very small degree of squeezing in the quadrature reflected within a restricted region of negative variance. On the other hand, the region within which CPT takes hold, around $\Delta_{a} / \gamma_{a} \approx 2.38$, is found to reduce 

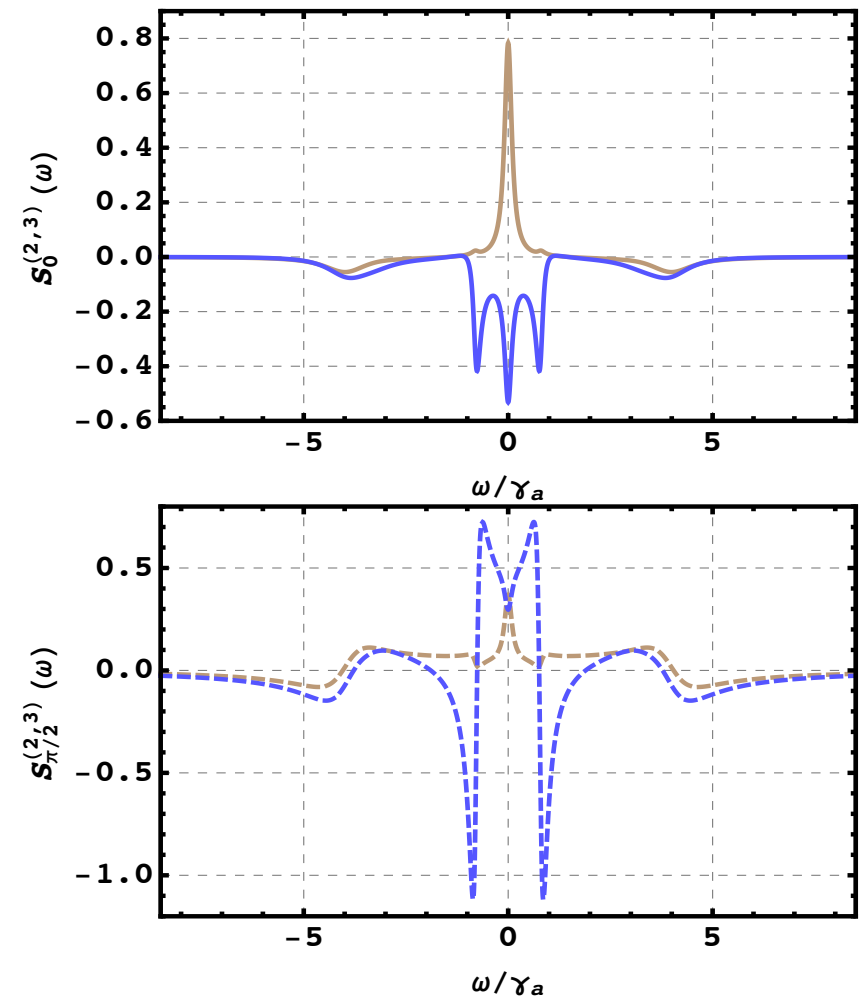

FIG. 9: Fourier cosine transform of $h_{\phi}^{(N)}(\tau \geq 0)$, Eq. (29), for $N=2$ (brown) and $N=3$ (blue). Upper and lower panels correspond, respectively, to the cases $\phi=0$ and $\pi / 2$. The parameters are the same as those of Fig. 5

fluctuations, approximately, to the extent of a coherent state. It was also verified that the in-phase quadrature (not shown) did not feature squeezed fluctuations in any parameter regime of the aforesaid transition.

\section{CONCLUSIONS}

Using the framework of conditional homodyne detection, we have analyzed the nearby effect of coherent population trapping on the phase-dependent quantum fluctuations, in both time and frequency domains, of the light fluoresced in the probe transition from a coherently driven $\Lambda$-type three-level atom. Given the feasibility of implementing the outlined optical system, a single ${ }^{138} \mathrm{Ba}^{+}$ion [3, 11], our findings are expected to bolster further experimental investigations to be benchmarked against CHD-based theoretical predictions.

It is worth underlying that the CHD framework proves to be a versatile tool to discern the contribution of phasedependent fluctuations of different orders, concluding that the light scattered under the aforesaid conditions is essentially non-Gaussian; i.e., the correlation of thirdorder in the dipole fluctuation operators prevails. NonGaussianity, notably, manifests in two main ways. On the one hand, the amplitude-intensity correlation is, in general, time-asymmetric, indicating that amplitude and
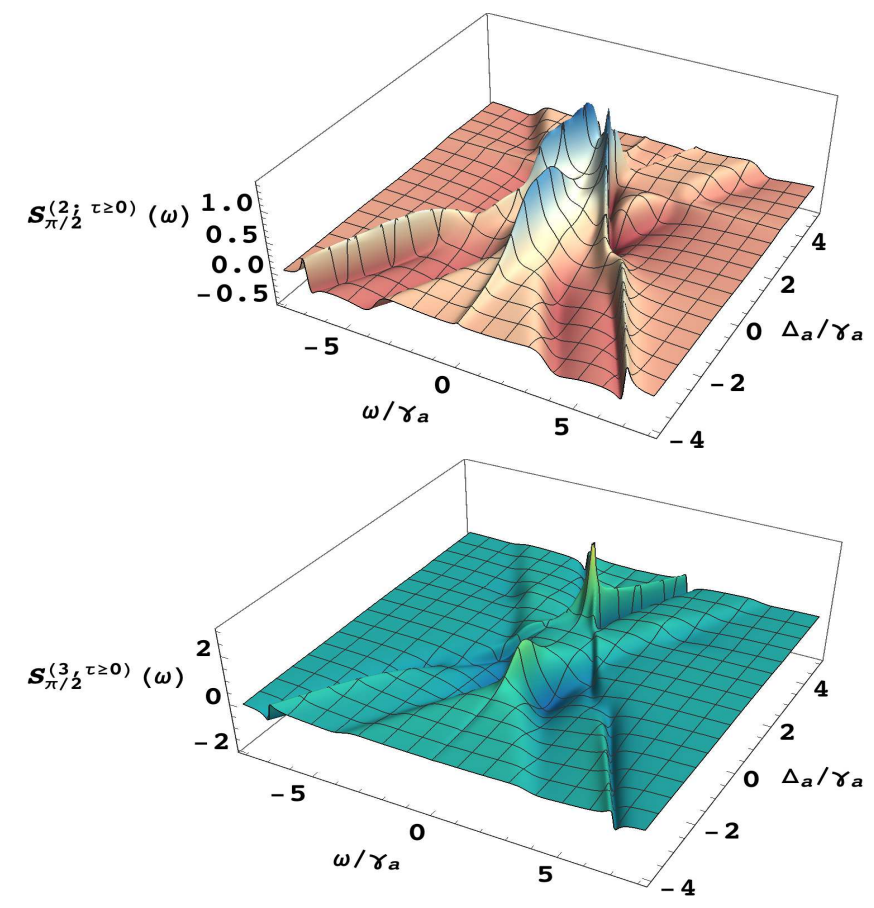

FIG. 10: Fourier transform of $h_{\pi / 2}^{(2,3)}(\tau \geq 0)$, as a function of the scaled detuning $\Delta_{a} / \gamma_{a}$, for $\Omega_{a} / \gamma_{a} \approx 1.12$. The set of parameters is the same as in Fig. 5

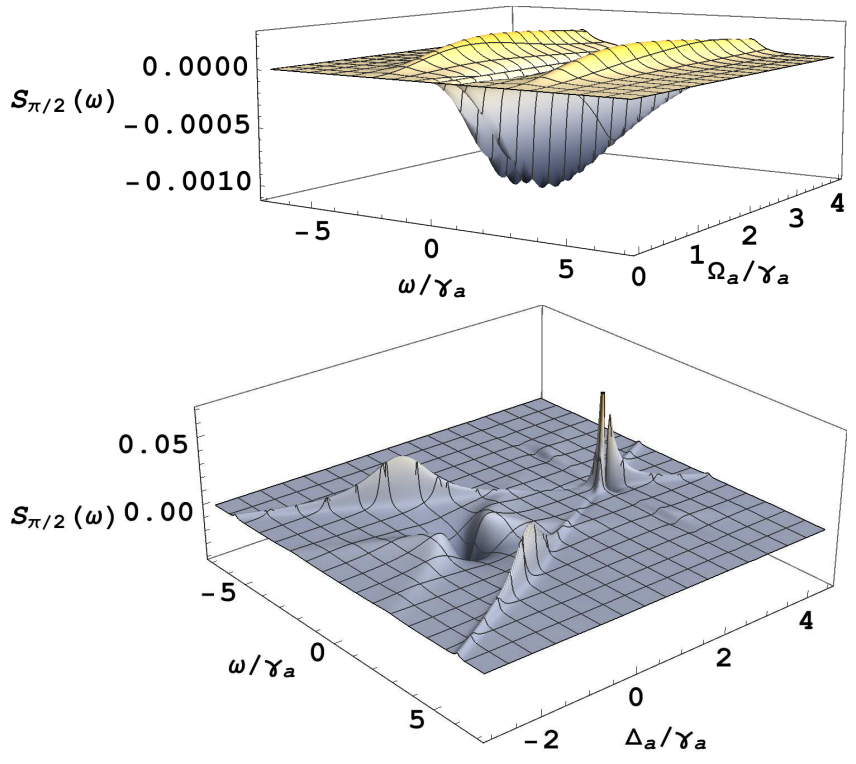

FIG. 11: Spectrum of squeezing of the $\phi=\pi / 2$ quadrature as a function of the probe field intensity (upper panel, for $\Delta_{a} / \gamma_{a}=2.38$ ) and detuning (lower panel, for $\Omega_{a} / \gamma_{a}=0.1$ ). For both, $\Omega_{b} \approx 2.15 \gamma_{a}$ and $\Delta_{b}=2.38 \gamma_{a}$.

intensity of the radiated field have different noise properties. On the other, the non-linearity imposed by a saturating excitation regime leads to fluctuations away from the ideal weak-field squeezing regime.

The role of CPT in CHD is explored with particular 


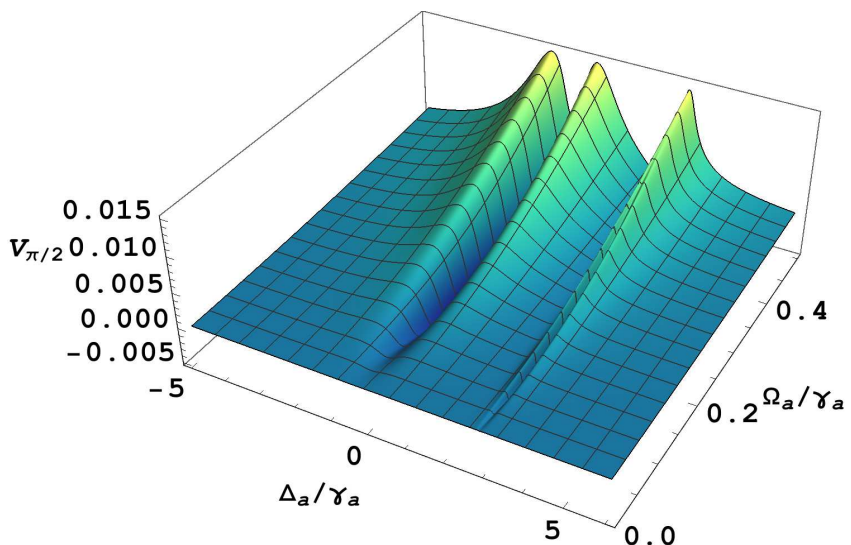

FIG. 12: Variance for $\phi=\pi / 2$, as a function of the scaled Rabi frequency $\Omega_{a} / \gamma_{a}$. For both, $\Omega_{b} \approx 2.15 \gamma_{a}$ and $\Delta_{b}=$ $2.38 \gamma_{a}$.

focus on the spectra of quadratures. In this regard, as a function of the probe detuning, the spectral content confirms once again the prevailing contribution of thirdorder fluctuations to the outcome of the measurements, for both quadratures. This fact is also reinforced by examining the variance (the integrated spectrum) of fluorescence.

\section{ACKNOWLEDGMENTS}

The authors thank Dr. Irán Ramos-Prieto for useful coversations and help with the figures.

\section{Appendix A: Correlations and Spectra}

Here, we succinctly describe the evaluation of the expectation values of two-time correlations and spectra used throughout this work.

From the equations of motion of the atomic operators, Eqs. (3) to (9), which can be put into the concise form $\dot{\mathbf{s}}(t)=\mathbf{M s}(t)$, with $\mathbf{s}=$ $\left\{\sigma_{e e}, \sigma_{a e}, \sigma_{b e}, \sigma_{e a}, \sigma_{a a}, \sigma_{b a}, \sigma_{e b}, \sigma_{a b}, \sigma_{b b}\right\}^{T}$ and $\mathbf{M}$ the parameter matrix (to be specified), together with the use of the quantum regression formula 32], we seek the general solution to the equation

$$
\partial_{\tau} \mathbf{g}(\tau)=\mathbf{M g}(\tau)
$$

where $\mathbf{g}(\tau)=\left\langle\Delta \sigma_{e a}(0) \Delta \mathbf{s}(\tau) \Delta A_{-}(0)\right\rangle_{s s}$ is the corresponding vector of correlation functions. For the secondorder correlations, $\Delta A_{-}=\mathbf{1}$; for the third-order ones, $\Delta A_{-}=\Delta \sigma_{a e}$. Its solution can be written in the form $\mathbf{g}(\tau)=e^{\mathbf{M} \tau} \mathbf{g}(0)$, where the initial condition $\mathbf{g}(0)=\mathbf{g}_{s s}$, given in terms of the steady state solution of populations and coherences, is solved numerically.

The incoherent spectrum requires, for instance, handling the time dependence of the correlation $\{\mathbf{g}(\tau)\}_{m}=$ $\left\langle\Delta \sigma_{e a}(0) \Delta \sigma_{a e}(\tau)\right\rangle_{s s}$, where the subindex $m$-th denotes the element of the vector to be taken. The present matrix analysis saves the work of solving the correlation explicitly followed by time integration, namely, for $\Delta A_{-}=1$,

$$
\begin{aligned}
S_{i n c}(\omega)= & \frac{1}{\pi \alpha_{e e}} \operatorname{Re}\left\{\int_{0}^{\infty} d \tau e^{-(i \omega \mathbf{1}-\mathbf{M}) \tau}\left\langle\Delta \sigma_{e a} \Delta \mathbf{s}\right\rangle_{s s}\right\}_{m} \\
= & \frac{1}{\pi \alpha_{e e}} \operatorname{Re}\left\{-\left.(i \omega \mathbf{1}-\mathbf{M})^{-1} e^{-(i \omega \mathbf{1}-\mathbf{M}) \tau}\right|_{0} ^{\infty}\right. \\
& \left.\times\left\langle\Delta \sigma_{e a} \Delta \mathbf{s}\right\rangle_{s s}\right\}_{m} \\
= & \frac{1}{\pi \alpha_{e e}} \operatorname{Re}\left\{(i \omega \mathbf{1}-\mathbf{M})^{-1}\left\langle\Delta \sigma_{e a} \Delta \mathbf{s}\right\rangle_{s s}\right\}_{m},
\end{aligned}
$$

where $\mathbf{1}$ is the $n \times n$ identity matrix. The spectra corresponding to the CHD correlations are calculated in the same manner, thus giving us the sought results

$$
\begin{aligned}
S_{\phi}^{(2)}(\omega)= & \left.\frac{2 \gamma_{a}}{\alpha_{\phi}} \operatorname{Re}\left\{\alpha_{a e} e^{-i \phi}\left[(i \omega \mathbf{1}-\mathbf{M})^{-1}-(i \omega \mathbf{1}+\mathbf{M})^{-1}\right) \mathbf{g}(0)\right]_{m}\right\} \\
& \left.+\frac{2 \gamma_{a}}{\alpha_{\phi}} \operatorname{Re}\left\{\alpha_{a e} e^{i \phi}\left[(i \omega \mathbf{1}-\mathbf{M})^{-1}-(i \omega \mathbf{1}+\mathbf{M})^{-1}\right) \mathbf{g}(0)\right]_{n}\right\}, \\
S_{\phi}^{(3)}(\omega)= & \left.\frac{2 \gamma_{a}}{\alpha_{\phi}} \operatorname{Re}\left\{e^{-i \phi}\left[(i \omega \mathbf{1}-\mathbf{M})^{-1}-(i \omega \mathbf{1}+\mathbf{M})^{-1}\right) \mathbf{g}(0)\right]_{p}\right\},
\end{aligned}
$$

for the second- and third-order fluctuations, respectively, and

$$
S_{j, \phi}^{(\tau \leq 0)}(\omega)=\frac{2 \gamma_{j}}{\alpha_{\phi}} \operatorname{Re}\left\{e^{-i \phi}\left[\left((i \omega \mathbf{1}-\mathbf{M})^{-1}-(i \omega \mathbf{1}+\mathbf{M})^{-1}\right) \mathbf{g}(0)\right]_{q}\right\}
$$

for fluctuations associated with negative time intervals. The elements of the vectors, denoted by subindexes $m, n, p$ and $q$, have to be chosen appropriately to match the corresponding correlation it seeks to assess.

For the sake of completeness, the initial conditions of the correlations $(\tau=0)$ are encapsulated by using the 
fluctuation operator approach as

$$
\begin{aligned}
\left\langle\Delta \sigma_{i j} \Delta \sigma_{k l}\right\rangle_{s s} & =\alpha_{i l} \delta_{j k}-\alpha_{i j} \alpha_{k l}, \\
\left\langle\Delta \sigma_{i g} \Delta \sigma_{j k} \Delta \sigma_{g i}\right\rangle_{s s} & =2\left|\alpha_{i g}\right|^{2} \alpha_{j k}+\alpha_{i i}\left(\delta_{g j} \delta_{k g}-\alpha_{j k}\right)-\alpha_{i k} \alpha_{g i} \delta_{g j}-\alpha_{i g} \alpha_{j i} \delta_{k g},
\end{aligned}
$$

for the second- and third-order fluctuations, respectively. More explicitly, for the $e \rightarrow a$ transition, they become

$$
\left\langle\Delta \sigma_{e a} \Delta \mathbf{s}\right\rangle_{s s}=\left\{-\alpha_{e a} \alpha_{e e}, \alpha_{e e}-\alpha_{e a} \alpha_{a e},-\alpha_{e a} \alpha_{b e},-\alpha_{e a}^{2}, \alpha_{e a}\left(1-\alpha_{a a}\right),-\alpha_{e a} \alpha_{b a}-\alpha_{e a} \alpha_{e b}, \alpha_{e b}-\alpha_{e a} \alpha_{a b},-\alpha_{e a} \alpha_{b b}\right\}^{T}
$$

and

$$
\left(\begin{array}{c}
\left\langle\Delta \sigma_{e a} \Delta \sigma_{e e} \Delta \sigma_{a e}\right\rangle_{s s} \\
\left\langle\Delta \sigma_{e a} \Delta \sigma_{a e} \Delta \sigma_{a e}\right\rangle_{s s} \\
\left\langle\Delta \sigma_{e a} \Delta \sigma_{b e} \Delta \sigma_{a e}\right\rangle_{s s} \\
\left\langle\Delta \sigma_{e a} \Delta \sigma_{e a} \Delta \sigma_{a e}\right\rangle_{s s} \\
\left\langle\Delta \sigma_{e a} \Delta \sigma_{a a} \Delta \sigma_{a e}\right\rangle_{s s} \\
\left\langle\Delta \sigma_{e a} \Delta \sigma_{b a} \Delta \sigma_{a e}\right\rangle_{s s} \\
\left\langle\Delta \sigma_{e a} \Delta \sigma_{e b} \Delta \sigma_{a e}\right\rangle_{s s} \\
\left\langle\Delta \sigma_{e a} \Delta \sigma_{a b} \Delta \sigma_{a e}\right\rangle_{s s} \\
\left\langle\Delta \sigma_{e a} \Delta \sigma_{b b} \Delta \sigma_{a e}\right\rangle_{s s}
\end{array}\right)=\left(\begin{array}{c}
\alpha_{e e}\left(2\left|\alpha_{e a}\right|^{2}-\alpha_{e e}\right) \\
-2 \alpha_{a e}\left(\alpha_{e e}-\left|\alpha_{e a}\right|^{2}\right) \\
\alpha_{b e}\left(2\left|\alpha_{e a}\right|^{2}-\alpha_{e e}\right) \\
2 \alpha_{e a}\left(\left|\alpha_{e a}\right|^{2}-\alpha_{e e}\right) \\
\left(2\left|\alpha_{e a}\right|^{2}-\alpha_{e e}\right)\left(\alpha_{a a}-1\right) \\
\alpha_{b a}\left(2\left|\alpha_{e a}\right|^{2}-\alpha_{e e}\right)-\alpha_{e a} \alpha_{b e} \\
\alpha_{e b}\left(2\left|\alpha_{e a}\right|^{2}-\alpha_{e e}\right) \\
\alpha_{a b}\left(2\left|\alpha_{e a}\right|^{2}-\alpha_{e e}\right)-\alpha_{e b} \alpha_{a e} \\
\alpha_{b b}\left(2\left|\alpha_{e a}\right|^{2}-\alpha_{e e}\right)
\end{array}\right) .
$$

[1] E. Arimondo, Progress in Optics, 35, 257 (1996).

[2] M. Fleischhauer, A. Imamoglu and J. P. Marangos, Rev. Mod. Phys. 77633 (2005).

[3] L. Slodička, G. Hétet, S. Gerber, M. Hennrich, and R. Blatt, Phys. Rev. A 105153604 (2010).

[4] J. Hwang, M. Pototschig, R. Lettow, G. Zumofen, A. Renn, S. Götzinger, and V. Sandoghdar, Nature (London) 460, 76 (2009).

[5] D. F. Walls and P. Zoller, Phys. Rev. Lett. 47709 (1981).

[6] M. J. Collett, D. F. Walls, P. Zoller, Optics Commun. 52, 145-149 (1984).

[7] C. H. H. Schulte, J. Hansom, A. E. Jones, C. Matthiesen, C. Le Gall, and M. Atatüre, Nature 525, 222 (2015).

[8] W. Vogel, Phys. Rev. Lett. 67, 2450-2452 (1991).

[9] W. Vogel, Phys. Rev. A 51, 4160 (1995).

[10] B. Kühn, W. Vogel, M. Mraz, S. Köhnke, and B. Hage, Phys. Rev. Lett. 118, 153601 (2017).

[11] S. Gerber, D. Rotter, L. Slodička, J. Eschner, H. J. Carmichael, and R. Blatt, Phys. Rev. Lett. 102, 183601 (2009).

[12] E. V. Shchukin and W. Vogel, Phys. Rev. A 72, 043808 (2005).

[13] E. V. Shchukin and W. Vogel, Phys. Rev. Lett. 96, 200403 (2006).

[14] H. J. Carmichael, H. M. Castro-Beltran, G. T. Foster, and L. A. Orozco, Phys. Rev. Lett. 85, 1855 (2000).

[15] G. T. Foster, L. A. Orozco, H. M. Castro-Beltran, and H. J. Carmichael, Phys. Rev. Lett. 85, 3149 (2000).

[16] For a review on CHD see H. J. Carmichael, G. T. Foster, L. A. Orozco, J. E. Reiner, and P. R. Rice, in Progress in Optics 46, E. Wolf, ed. (Elsevier, 2004).

[17] H. M. Castro-Beltran, Opt. Commun. 283, 4680 (2010).

[18] H. M. Castro-Beltran, L. Gutierrez, and L. Horvath,
Appl. Math. Inf. Sci. 9, 2849 (2015).

[19] A. Denisov, H. M. Castro-Beltran, and H. J. Carmichael, Phys. Rev. Lett. 88, 243601 (2002).

[20] H. M. Castro-Beltran, R. Roman-Ancheyta, and L. Gutierrez, Phys. Rev. A, 93, 033801 (2016).

[21] E. R. Marquina-Cruz and H. M. Castro-Beltran, Laser Phys. 18, 157 (2008).

[22] L. Gutierrez, H. M. Castro-Beltran, R. Roman-Ancheyta, and L. Horvath, J. Opt. Soc. Am. B 34, 2301 (2017).

[23] Q. Xu, E. Greplova, B. Julsgaard, and K. Mølmer, Phys. Scripta 90, 128004 (2015).

[24] Q. Xu and K. Mølmer, Phys. Rev. A 92, 033830 (2015).

[25] S. Gammelmark, B. Julsgaard, and K. Mølmer, Phys. Rev. Lett. 111, 160401 (2013).

[26] F. Wang, X. Feng, and C. H. Oh, Laser Phys. Lett. 13, 105201 (2016).

[27] O. de los Santos-Sánchez. Front. Phys. 14, 61601 (2019).

[28] T. Zhao, Z.-A. Peng, G.-Q. Yang, G.-M. Huang, and G.X. Li, Optics Express 28, 379 (2020).

[29] E. A. M. Nuñez-Portela, A. T. Grier, K. Jungmann, A. Mohanty, N. Valappol, and L. Willmann, Phys. Rev. A, 91, 060501(R) (2015).

[30] Y. Stalgies, I. Siemers, B. Appasamy, T. Altevogt, and P. E. Toscheck, Europhys. Lett. 35, 259 (1996).

[31] C. Cohen-Tannoudji, J. Dupont-Roc, and G. Grynberg, Atom-Photon Interactions: Basic Processes and Applications (Wiley, New York, 1992).

[32] H. J. Carmichael, Statistical Methods in Quantum Optics 1: Master Equations and Fokker-Planck Equations (Springer-Verlag, Berlin, 2002).

[33] H. J. Carmichael, J. Opt. Soc. Am. B 4, 1588 (1987). 